\title{
Indirect adaptive regulation strategy for the attenuation of time varying narrow-band disturbances applied to a benchmark problem
}

\author{
Tudor-Bogdan Airimitgoaie ${ }^{1,2}$, Abraham Castellanos Silva ${ }^{1}$, and Ioan Doré Landau ${ }^{1}$
}

\begin{abstract}
The paper presents an indirect adaptive regulation algorithm for the attenuation of unknown narrow-band disturbances. The main features of this new scheme are: (i) the use of adaptive Band-stop Filters (BSFs) tuned at the frequencies of the disturbance and (ii) a procedure for direct identification of frequencies contained in the disturbance. The use of adaptive BSFs allows to introduce the desired attenuation of the disturbance (instead of total rejection) and simplifies the shaping of the output sensitivity function (to meet the specification for the tolerated amplification outside the frequencies of the disturbance). The proposed approach is evaluated on the benchmark simulator and on the benchmark active vibration control system.
\end{abstract}

Index Terms-Indirect adaptive regulation, active vibration control, inertial actuators, multiple narrow-band disturbances

\section{INTRODUCTION}

$\mathbf{T}$ HE present benchmark concerns attenuation of unknown and time varying narrow-band disturbances without an explicit measurement of the disturbance [13]. Only the residual force measurement is provided. Therefore a feedback approach has to be considered for disturbance attenuation. In general, one considers the disturbances as being a white noise or a Dirac impulse passed through a filter which characterises the model of the disturbance. For the purpose of this paper, the disturbances are considered to be unknown and/or time varying multiple narrow-band disturbances, in other words their model has time varying coefficients. Adaptive feedback control methods can then be used either in a direct scheme that updates the parameters of a controller at each sampling time or when the disturbance changes or in an indirect scheme that treats the problems of disturbance estimation and controller updating separately.

Various design procedures have been described in the scientific literature: (i) the internal model principle (IMP) ([11], [2], [14]), (ii) the disturbance observer ([19], [9]), and (iii) the use of the phase-locked loop structure ([7], [6]). A popular methodology for this adaptive regulation problem is the design of a controller that incorporates the model of the disturbance (internal model principle). Using the Youla-Kučera parametrization of the controller a direct adaptation technique can be implemented. Using the IMP principle, the complete rejection of the disturbances is attempted (asymptotically). In the case of several narrow-band disturbances, the "water bed" effect on the output sensitivity function (amplification introduced at the other frequencies than those of the disturbances)

\footnotetext{
${ }^{1}$ Control systems department of GIPSA-lab, St. Martin d'Héres, 38402 FRANCE (e-mail: [tudor-bogdan.airimitoaie, abraham.castellanos-silva, ioandore.landau]@gipsa-lab.grenoble-inp.fr).

${ }^{2}$ Faculty of Automatic Control and Computers, University "Politehnica" of Bucharest, Bucharest, 060042 ROMANIA (e-mail: tudor.airimitoaie@acse.pub.ro).
}

using IMP may become unacceptable in terms of performance as well as in terms of robustness (unacceptable profile of the output sensitivity function).

In practice however, and in particular for the present benchmark, we do not need a complete rejection of the narrowband disturbances but just a level of attenuation (IMP does too much!). Introducing only a level of attenuation combined with an appropriate controller design will reduce the "water bed" effect on the output sensitivity function improving both robustness and performance (by reducing the unwanted amplification of the noise). This will become particularly useful in the case of multiple narrow-band disturbances.

In this paper, an indirect adaptive regulation method is presented that is capable of introducing a desired level of attenuation on the disturbances. The most important advantage of this scheme is that the loss of robustness due to the "water bed" effect on the Bode integral of the output sensitivity function can be easily controlled by the design parameters of the new controller. The proposed procedure is based on the shaping of the output sensitivity function using band stop-filters (BSFs) centred at the frequencies corresponding to spikes in the spectrum of the disturbance. One interesting fact that should be mentioned is that the zeros of these BSFs are implemented in the controller while their poles are introduced as desired poles of the closed loop (see also [24], [16]). Reduction of the complexity of the computations has been achieved by considering the Youla-Kučera (YK) parametrization of the controller ([31], [10], [29]). This is very important in the perspective of using this controller design procedure in an adaptive scheme. It is important to underline that previous approaches for indirect adaptive regulation were still based on the use of the IMP and the identification of a model of the disturbance was enough for implementing the procedure ([14]).

In order to use adaptive BSFs for disturbance attenuation, it is necessary to estimate the frequencies of the narrowband signals in the disturbance. Therefore a procedure for the direct estimation of the frequencies of the disturbance has been implemented. Several methods have been proposed by the signal processing community for solving the problem of frequencies estimation from a narrow-band signal ([30]). In a continuous time framework and for a small number of disturbances recent solutions have been described in [20], [4], [5], [25]. For estimation using discrete time signals and a theoretically unlimited number of narrow-band spikes, the adaptive notch filter (ANF) approach has been proposed in [22], [21] and analysed in a statistical framework in [28]. Revised and improved versions have also been proposed in a number of articles [26], [27], [8], [18], [12], [23]. In this paper, the estimation approach presented in [28], [21] will be used. 
Combining the frequency estimation procedure and the control design procedure, an indirect adaptive regulation system for attenuation of multiple unknown and/or time varying narrowband disturbances is obtained, which will be denoted IBSF in the remainder of this paper.

The paper is organised as follows. Section II presents the general plant and controller structure in the context of the YK parametrization. To better understand the proposed approach, the linear controller design is presented in Section III considering, temporarily, constant and known frequencies of the narrow-band disturbances. Then in Section IV the frequency estimation technique based on ANF is recalled which can be combined with the linear controller design technique from the previous section to complete the indirect adaptive controller scheme. Section V discusses briefly the design of the central controller and simulation results are shown in Section VI. Experimental results are given in Section VII where also a comparison with simulation results is performed. Concluding remarks are presented in Section VIII.

\section{Plant Representation And Controller STRUCTURE}

The structure of the LTI discrete time model of the plant, also called secondary path, used for controller design is

$$
G\left(z^{-1}\right)=\frac{z^{-d} B\left(z^{-1}\right)}{A\left(z^{-1}\right)}=\frac{z^{-d-1} B^{*}\left(z^{-1}\right)}{A\left(z^{-1}\right)},
$$

where

$$
\begin{aligned}
A\left(z^{-1}\right) & =1+a_{1} z^{-1}+\cdots+a_{n_{A}} z^{-n_{A}} \\
B\left(z^{-1}\right) & =b_{1} z^{-1}+\cdots+b_{n_{B}} z^{-n_{B}}=z^{-1} B^{*}\left(z^{-1}\right), \\
B^{*}\left(z^{-1}\right) & =b_{1}+\cdots+b_{n_{B}} z^{-n_{B}+1}
\end{aligned}
$$

and $d$ is the plant pure time delay in number of sampling periods ${ }^{1}$. In the context of this paper the hypothesis of constant dynamic characteristics of the AVC system is made and it is also supposed that the corresponding control model (secondary path) is accurately identified from input/output data.

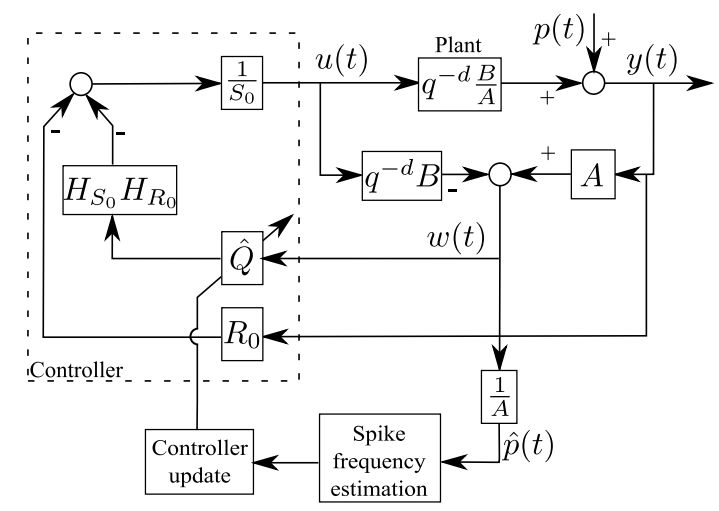

Fig. 1. Indirect adaptive regulation scheme using Youla-Kučera parametrized controller with adaptive $\hat{Q}$ filter.

${ }^{1}$ The complex variable $z^{-1}$ will be used to characterise the system's behaviour in the frequency domain and the delay operator $q^{-1}$ will be used for the time domain analysis.
The output of the plant $y(t)$ and the input $u(t)$ may be written as (see Fig. 1):

$$
\begin{aligned}
y(t) & =\frac{q^{-d} B\left(q^{-1}\right)}{A\left(q^{-1}\right)} \cdot u(t)+p(t), \\
S\left(q^{-1}\right) \cdot u(t) & =-R\left(q^{-1}\right) \cdot y(t),
\end{aligned}
$$

with $p(t)=\frac{N_{p}}{D_{p}} \delta(t)+v(t)$, where $\delta(t)$ is the Dirac impulse passed through a model of the primary path, whose denominator, $D_{p}$, has all its zeroes on the unit circle, and $v(t)$ is a zero mean white noise.

In this paper, the Youla-Kučera parametrization ([3], [31]) is used. Supposing a generalized infinite impulse response (IIR) representation of the adaptive $\mathrm{Q}$ filter

$$
Q\left(z^{-1}\right)=\frac{B_{Q}\left(z^{-1}\right)}{A_{Q}\left(z^{-1}\right)},
$$

the controller's polynomials are ${ }^{2}$ :

$$
\begin{aligned}
& R=R_{0} A_{Q}+A B_{Q} H_{S_{0}} H_{R_{0}}, \\
& S=S_{0} A_{Q}-z^{-d} B B_{Q} H_{S_{0}} H_{R_{0}} .
\end{aligned}
$$

where $R_{0}$ and $S_{0}$ define the central controller and have the expressions:

$$
\begin{aligned}
& S_{0}=1+s_{1}^{0} z^{-1}+\ldots+s_{n_{S}}^{0} z^{-n_{S}}=S_{0}^{\prime} \cdot H_{S_{0}}, \\
& R_{0}=r_{0}^{0}+r_{1}^{0} z^{-1}+\ldots+r_{n_{R}}^{0} z^{-n_{R}}=R_{0}^{\prime} \cdot H_{R_{0}} .
\end{aligned}
$$

Let define also the characteristic polynomial of the nominal system,

$$
P_{0}\left(z^{-1}\right)=A\left(z^{-1}\right) S_{0}\left(z^{-1}\right)+z^{-d} B\left(z^{-1}\right) R_{0}\left(z^{-1}\right),
$$

which specifies the desired closed loop poles of the feedback loop composed only by the process and the central controller (see also [16]). The characteristic polynomial of the closed loop with Youla-Kučera parametrized controller becomes

$$
P\left(z^{-1}\right)=A_{Q}\left(z^{-1}\right) P_{0}\left(z^{-1}\right) \text {. }
$$

In (10) and (11), $H_{S_{0}}\left(z^{-1}\right)$ and $H_{R_{0}}\left(z^{-1}\right)$ represent prespecified parts of the controller (used for example to incorporate the internal model of a disturbance or to open the loop at certain frequencies) and $S_{0}^{\prime}\left(z^{-1}\right)$ and $R_{0}^{\prime}\left(z^{-1}\right)$ are computed. The central controller is designed in order to fulfil desired specifications in the absence of the disturbance.

We define the output sensitivity function (the transfer function between the disturbance $p(t)$ and the output of the system $y(t))$ as

$$
S_{y p}\left(z^{-1}\right)=\frac{A\left(z^{-1}\right) S\left(z^{-1}\right)}{P\left(z^{-1}\right)}
$$

and the input sensitivity function (the transfer function between the disturbance $p(t)$ and the control input $u(t))$ as

$$
S_{u p}\left(z^{-1}\right)=-\frac{A\left(z^{-1}\right) R\left(z^{-1}\right)}{P\left(z^{-1}\right)} .
$$

It is important to remark that one should only reject disturbances located in frequency regions where the plant model

\footnotetext{
${ }^{2}$ The argument $\left(z^{-1}\right)$ will be omitted in some of the following equations to make them more compact.
} 
has enough gain. This can be seen by looking at eq. (14) and noticing that perfect rejection at a certain frequency, $\omega_{0}$, is obtained iff $S\left(e^{-j \omega_{0}}\right)=0$. On the other hand, from eq. (15) one can see that this has a bad effect on the control input if the gain of the secondary path is too small at $\omega_{0}$, since at this frequency the modulus of the input sensitivity function becomes $\left|S_{u p}\left(e^{-j \omega_{0}}\right)\right|=\left|\frac{A\left(e^{-j \omega_{0}}\right)}{B\left(e^{-j \omega_{0}}\right)}\right|$. This implies that the robustness vs additive plant model uncertainties is reduced and the stress on the actuator will become important. Furthermore, it can be observed that serious problems will occur if $B\left(z^{-1}\right)$ has complex zeros close to the unit circle at frequencies where an important attenuation of disturbances is introduced. It is mandatory to avoid attenuation of disturbances at these frequencies ([14]).

In addition to what has already been specified, it is also important to have a low magnitude of the input sensitivity function outside the region of attenuation in order to avoid amplification of noise and to have a good robustness with respect to model uncertainties.

\section{INDIRECT AdAPTIVE REgulation BASED ON BSFS FOR DisturbancE ATTENUATION}

In this section, a technique of output sensitivity function shaping for narrow-band disturbance attenuation is presented. The controllers parameters computation procedure will be presented considering constant and known frequencies of the narrow-band disturbances $\omega_{i}, \forall i \in\{1, \ldots, n\}$ and $n$ (number of spikes in the disturbance's spectral characteristic) is also known (a technique for estimating the frequencies is presented in Section IV).

The design uses BSFs to shape the output sensitivity function. Following [16], [24], there exist digital filters $\frac{H_{S_{i}}}{P_{F_{i}}}$, which will assure the desired attenuation of a narrow-band disturbance. The numerators of these filters are directly included in the controller. The denominators specify a factor in the desired closed loop characteristic polynomial. The transfer function of the BSFs is

$$
\frac{S_{B S F_{i}}\left(z^{-1}\right)}{P_{B S F_{i}}\left(z^{-1}\right)}=\frac{1+\beta_{1}^{i} z^{-1}+\beta_{2}^{i} z^{-2}}{1+\alpha_{1}^{i} z^{-1}+\alpha_{2}^{i} z^{-2}},
$$

resulting from the discretization of a continuous filter (see also [24], [16])

$$
F_{i}(s)=\frac{s^{2}+2 \zeta_{n_{i}} \omega_{i} s+\omega_{i}^{2}}{s^{2}+2 \zeta_{d_{i}} \omega_{i} s+\omega_{i}^{2}}
$$

using the bilinear transformation. This filter introduces an attenuation of

$$
M_{i}=-20 \cdot \log _{10}\left(\frac{\zeta_{n_{i}}}{\zeta_{d_{i}}}\right)
$$

at the frequency $\omega_{i}$. Positive values of $M_{i}$ denote attenuations $\left(\zeta_{n_{i}}<\zeta_{d_{i}}\right)$ and negative values denote amplifications $\left(\zeta_{n_{i}}>\right.$ $\left.\zeta_{d_{i}}\right)^{3}$. For $n$ narrow-band disturbances, $n$ BSFs will be used

$$
H_{B S F}\left(z^{-1}\right)=\frac{S_{B S F}\left(z^{-1}\right)}{P_{B S F}\left(z^{-1}\right)}=\frac{\prod_{i=1}^{n} S_{B S F_{i}}\left(z^{-1}\right)}{\prod_{i=1}^{n} P_{B S F_{i}}\left(z^{-1}\right)} .
$$

\footnotetext{
${ }^{3}$ For frequencies below $0.17 f_{s}$ ( $f_{s}$ is the sampling frequency) the design can be done with a very good precision directly in discrete time ([16]).
}

Remark: The design parameters for each BSF are the desired attenuation $\left(M_{i}\right)$, the central frequency of the filter $\left(\hat{\omega}_{i}\right)$ and the damping of the denominator $\left(\zeta_{d_{i}}\right)$. The denominator damping is used to adjust the frequency bandwidth of the BSF. For very small values of the frequency bandwidth the influence of the filters on frequencies other than those defined by $\hat{\omega}_{i}$ is negligible. Therefore, the number of BSFs and subsequently that of the narrow-band disturbances that can be compensated can be as large as necessary ${ }^{4}$.

Next, the computation of the controller's $S\left(z^{-1}\right)$ and $R\left(z^{-1}\right)$ polynomials (eqs. (8) and (9)) is described taking into account that at the end the BSFs have to become part of the output sensitivity function. Without considering the Youla-Kučera parametrization, the controller is computed as solution of a Bezout equation $P\left(z^{-1}\right)=A\left(z^{-1}\right) S\left(z^{-1}\right)+z^{-d} B\left(z^{-1}\right) R\left(z^{-1}\right)$, where

$$
R\left(z^{-1}\right)=H_{R}\left(z^{-1}\right) R^{\prime}\left(z^{-1}\right), S\left(z^{-1}\right)=H_{S}\left(z^{-1}\right) S^{\prime}\left(z^{-1}\right),
$$

and $P\left(z^{-1}\right)$ is given by

$$
P\left(z^{-1}\right)=P_{0}\left(z^{-1}\right) P_{B S F}\left(z^{-1}\right) .
$$

In the last equation, $P_{B S F}$ is the combined denominator of all the BSFs, (19), and $P_{0}$ can define any other poles, e.g., from an initial robust control design as in (12). The fixed part of the controller denominator $H_{S}$ is in turn factorized into

$$
H_{S}\left(z^{-1}\right)=S_{B S F}\left(z^{-1}\right) H_{S_{0}}\left(z^{-1}\right)
$$

where $S_{B S F}$ is the combined numerator of the BSFs, (19), and $H_{S_{0}}$ is the fixed part of the denominator in the initial robust controller (see (10)). The fixed part of $R$ is equal to that used for the initial robust controller, i.e. $H_{R}=H_{R_{0}}$. It is easy to see that the output sensitivity function becomes

$$
S_{y p}\left(z^{-1}\right)=\frac{A\left(z^{-1}\right) S\left(z^{-1}\right)}{P\left(z^{-1}\right)}=\frac{A S^{\prime} H_{S_{0}} S_{B S F}}{P_{0} P_{B S F}}
$$

and the shaping effect of the BSFs upon the sensitivity functions is obtained.

The unknowns $S^{\prime}$ and $R^{\prime}$ are solutions of

$$
\begin{gathered}
P\left(z^{-1}\right)=P_{0}\left(z^{-1}\right) P_{B S F}\left(z^{-1}\right)=A\left(z^{-1}\right) H_{S}\left(z^{-1}\right) S^{\prime}\left(z^{-1}\right)+ \\
+z^{-d} B\left(z^{-1}\right) H_{R_{0}}\left(z^{-1}\right) R^{\prime}\left(z^{-1}\right)
\end{gathered}
$$

and can be computed by putting (24) into matrix form (see also [16]). The size of the matrix equation that needs to be solved is given by

$$
n_{B e z}=n_{A}+n_{B}+d+n_{H_{S_{0}}}+n_{H_{R_{0}}}+2 \cdot n-1,
$$

where $n_{A}, n_{B}$, and $d$ are respectively the order of the plant's model denominator, numerator, and delay (given in (2) and (3)), $n_{H_{S_{0}}}$ and $n_{H_{R_{0}}}$ are the orders of $H_{S_{0}}\left(z^{-1}\right)$ and $H_{R_{0}}\left(z^{-1}\right)$ respectively and $n$ is the number of narrow-band disturbances. Eq. (24) has an unique minimal degree solution for $S^{\prime}$ and $R^{\prime}$, if

$$
n_{P} \leq n_{B e z},
$$

${ }^{4}$ Of course, there is a compromise between the attenuation imposed and the number of narrow-band disturbances. 
where $n_{P}$ is the order of the pre-specified characteristic polynomial $P\left(q^{-1}\right)$. Also, it can be seen from (24) and (22) that the minimal orders of $S^{\prime}$ and $R^{\prime}$ will be:

$$
n_{S^{\prime}}=n_{B}+d+n_{H_{R_{0}}}-1, \quad n_{R^{\prime}}=n_{A}+n_{H_{S_{0}}}+2 \cdot n-1 .
$$

Note that for real time applications, the diophantine equation (24) has to be solved either at each sampling time (adaptive operation) or each time when a change in the narrowband disturbances' frequencies occurs (self-tuning operation).

The computational complexity related to the Bezout equation (24) is significant. We show next how the computation load of the algorithm can be reduced by the use of the YoulaKučera parametrization with IIR parameter (7). Using this, the initial robust controller from (10) and (11) becomes the central controller of the parametrization as in (8) and (9).

In (8) and (9), $A_{Q}\left(z^{-1}\right)$ will be chosen as the cumulated denominator of the BSFs, $P_{B S F}\left(z^{-1}\right)$, while $B_{Q}\left(z^{-1}\right)$ is computed so that it allows to introduce the BSFs' numerators into the fixed part of $S\left(z^{-1}\right)$, as in (22). Taking into account (9), this is equivalent to finding $B_{Q}\left(z^{-1}\right)$ from the Bezout equation

$$
S_{0}^{\prime} P_{B S F}=S_{B S F} S^{\prime}+q^{-d} B H_{R_{0}} B_{Q},
$$

where the common term $H_{S_{0}}\left(z^{-1}\right)$ has been eliminated.

In the last equation, the left side of the equal sign is known and on its right side only $S^{\prime}\left(z^{-1}\right)$ and $B_{Q}\left(z^{-1}\right)$ are unknown. This is also a Bezout equation which can be solved by finding the solution to a matrix equation of dimension

$$
n_{B e z_{Y K}}=n_{B}+d+n_{H_{R_{0}}}+2 \cdot n-1 \text {. }
$$

As it can be observed, the size of the new Bezout equation is reduced in comparison to (25) by $n_{A}+n_{H_{S_{0}}}$. For systems with large dimensions, this has a significant influence on the computation time (in Sections VI and VII, $n_{A}=22$ and $n_{H_{S_{0}}}=$ $0)$. Taking into account that the central controller is an unique and minimal degree solution of the Bezout equation (12), we find that the left hand side of (28) is a polynomial of degree

$$
n_{S_{0}^{\prime}}+2 \cdot n=2 \cdot n+n_{B}+d+n_{H_{R_{0}}}-1
$$

which is equal to the quantity given in (29). Therefore, the solution of the simplified Bezout equation (28) is unique and of minimal degree. Furthermore, the order of the $B_{Q}$ FIR filter is equal to $2 \cdot n-1$ (where $n$ is the number of narrow-band signals in the disturbance).

\section{Frequency Estimation Using Adaptive Notch FILTERS}

In order to use the proposed control strategy in the presence of unknown and/or time varying narrow-band disturbances, one needs an estimation in real time of the spikes' frequencies in the spectrum of the disturbance. In the framework of narrow-band disturbance rejection, it is usually supposed that the disturbances are in fact sinusoidal signals with variable frequencies. As specified in the introduction, it is assumed that the number of narrow-band disturbances $n$ is known (similar to [15], [14], [9]). A technique based on ANFs will be used to estimate the frequencies of the sinusoidal signals in the disturbance (more details can be found in [22], [21]). Under the hypothesis that the plant model parameters are constant and that an accurate identification experiment can be run, a reliable estimate $\hat{p}(t)$ of the disturbance signal can be obtained by using the disturbance observer

$$
\hat{p}(t+1)=y(t+1)-q^{-d} \frac{B^{*}\left(q^{-1}\right)}{A\left(q^{-1}\right)} u(t) .
$$

The signal $\hat{p}(t)$ can then be used to estimate the spike frequencies $\left(\hat{\omega}_{i}\right)$ with adaptive notch filters (ANF) as will be described in Section IV.

The general form of an ANF is

$$
H_{f}\left(z^{-1}\right)=\frac{A_{f}\left(z^{-1}\right)}{A_{f}\left(\rho z^{-1}\right)},
$$

where the polynomial $A_{f}\left(z^{-1}\right)$ is such that the zeros of the transfer function $H_{f}\left(z^{-1}\right)$ lie on the unit circle. A necessary condition for a monic polynomial to satisfy this property is that its coefficients have a mirror symmetric form

$$
A_{f}=1+a_{1}^{f} z^{-1}+\ldots+a_{n}^{f} z^{-n}+\ldots+a_{1}^{f} z^{-2 n+1}+z^{-2 n} .
$$

Another requirement is that the poles of the ANF should be on the same radial lines as the zeros but slightly closer to the origin of the unit circle. Using filter denominators of the general form $A_{f}\left(\rho z^{-1}\right)$ with $\rho$ a positive real number smaller but close to 1 , the poles have the desired property and are in fact located on a circle of radius $\rho$ ([22]).

The estimation algorithm will be detailed next. It is assumed that the disturbance signal (or a good estimation) is available. A cascade construction of second order ANF filters is considered. Their number is given by the number of narrow-band signals whose frequencies have to be estimated. The main idea behind this algorithm is to consider the signal $\hat{p}(t)$ as having the form

$$
\hat{p}(t)=\sum_{i=1}^{n} c_{i} \sin \left(\omega_{i} \cdot t+\beta_{i}\right)+v(t)
$$

where $v(t)$ is a noise affecting the measurement.

The ANF cascade form will be given by (this is an equivalent representation of eqs. (32) and (33))

$$
H_{f}\left(z^{-1}\right)=\prod_{i=1}^{n} H_{f}^{i}\left(z^{-1}\right)=\prod_{i=1}^{n} \frac{1+a^{f_{i}} z^{-1}+z^{-2}}{1+\rho a^{f_{i}} z^{-1}+\rho^{2} z^{-2}} .
$$

Next, the estimation of one spike's frequency is considered, assuming convergence of the other $n-1$, which can thus by filtered out of the estimated disturbance signal, $\hat{p}(t)$, by applying

$$
\hat{p}^{j}(t)=\prod_{\substack{i=1 \\ i \neq j}}^{n} \frac{1+a^{f_{i}} z^{-1}+z^{-2}}{1+\rho a^{f_{i}} z^{-1}+\rho^{2} z^{-2}} \hat{p}(t) .
$$

The prediction error is obtained from

$$
\varepsilon(t)=H_{f}\left(z^{-1}\right) \hat{p}(t)
$$

and can be computed based on one of the $\hat{p}^{j}(t)$ to reduce the computation complexity. Each cell can be adapted independently after prefiltering the signal by the others. Following 
the Recursive Prediction Error (RPE) technique, the gradient is obtained as

$$
\Psi^{j}(t)=-\frac{\partial \varepsilon(t)}{\partial a^{f_{j}}}=\frac{(1-\rho)\left(1-\rho z^{-2}\right)}{1+\rho a^{f_{j}} z^{-1}+\rho^{2} z^{-2}} \hat{p}^{j}(t) .
$$

The parametric adaptation algorithm can be summarised as

$$
\begin{aligned}
\hat{a}^{f_{j}}(t) & =\hat{a}^{f_{j}}(t-1)+F(t-1) \cdot \Psi^{j}(t) \cdot \varepsilon(t) \\
F(t) & =\frac{F(t-1)}{\lambda+F(t-1) \Psi^{j}(t)^{2}} .
\end{aligned}
$$

where $\hat{a}^{f_{j}}$ are estimations of the true $a^{f_{j}}$, which are connected to the narrow-band signals' frequencies by $\omega_{f_{j}}=$ $f_{s} \cdot \arccos \left(-\frac{a^{f_{j}}}{2}\right)$, where $f_{s}$ is the sampling frequency.

Combining the linear controller design presented in Section III with the spike frequency estimations presented here, an indirect adaptive regulation scheme is obtained. A stability proof for this scheme has been given in [1].

\section{Central Controller Design}

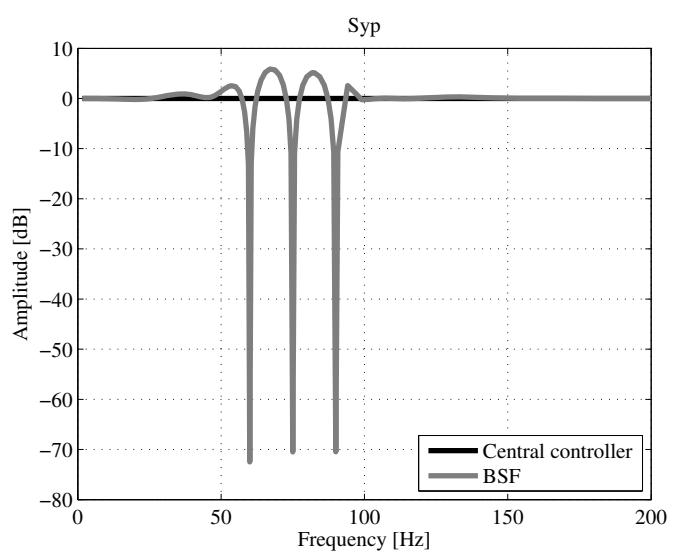

Fig. 2. Output sensitivity functions comparison for a central controller and a BSF controller designed to attenuate disturbances at 60, 75, and $90 \mathrm{~Hz}$.

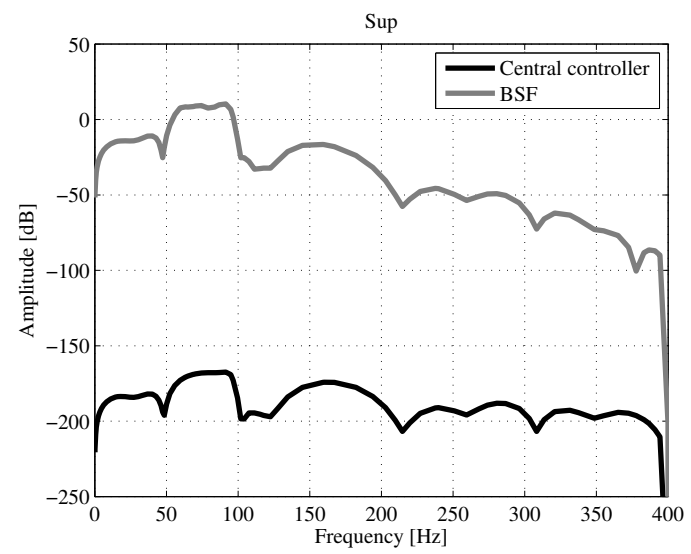

Fig. 3. Input sensitivity functions comparison for a central controller and a BSF controller designed to attenuate disturbances at 60, 75, and $90 \mathrm{~Hz}$.

A key element of the IBSF is the central controller, which is presented in eqs. (10) and (11). Its aim is to ensure closed loop robustness with respect to model uncertainties and noise outside the attenuation region. The design of the central controller is described in this section. The main tool used has been the sensitivity functions shaping. As there exist uncertainties in the estimated parameters of the system, an important aspect is that of minimising the effect of possibly undesired dynamics and unmodelled noise ([17]). Therefore, outside of the frequency region of interest for control (the disturbances are located between 50 and $95 \mathrm{~Hz}$ ) the input sensitivity (15) function is reduced.

For the specific case that was the benchmark active vibration control system, first all the poles of the secondary path are conserved as poles of the closed loop (the system is stable). In addition, two pairs of complex fixed auxiliary low damped poles are introduced near to the limits of the frequency region of interest, at 50 and at $95 \mathrm{~Hz}$, in order to improve the system's robustness. The effect is the decrease of the magnitudes of the sensitivity functions outside this region.

Finally, fixed parts are introduced in the central controller's numerator, $R_{0}\left(z^{-1}\right)$, for opening the loop at $0 f_{s}$ and $0.5 f_{s}$ (required by the benchmark specifications). No fixed parts were considered for $S_{0}\left(z^{-1}\right)$.

Given the characteristics of the BSFs, the design of the central controller is simplified since the shape of the sensitivity functions (i.e., the robustness of the closed loop system) is only slightly modified when the BSFs for attenuation are introduced. Therefore, a single central controller can be designed for the three levels of the benchmark.

Fig. 2 shows a comparison of the output sensitivity functions obtained with the central controller presented previously and a controller that introduces in addition $3 \mathrm{BSFs}$ to attenuate narrow-band disturbances at 60,75 , and $90 \mathrm{~Hz}$. For the BSFs, an attenuation of $80 \mathrm{~dB}$ and a damping of the denominator of 0.09 have been used. It can be observed that only a minor increase in the output sensitivity function is introduced for the desired level of attenuation, which proves that the proposed method is capable of offering satisfactory robust performance. For the same situation, the input sensitivity function is shown in Fig. 3. It can be seen that the transfer from disturbance to control signal is significantly below $0 \mathrm{~dB}$ outside the frequency region of interest even when the BSFs are introduced. As a consequence, the residual noise is not amplified and a good robustness with respect to plant model uncertainties is assured.

\section{Simulation Results}

Regarding the parameters used in the IBSF, through all the simulation and experimental tests, the ANFs use a $\rho$ of 0.92 and the BSFs have been chosen with an attenuation of $80 \mathrm{~dB}$ and a denominator damping of 0.09 .

The IBSF approach was tested first in simulation and the results are presented in the following subsections. The benchmark considers three levels in terms of number of unknown narrow-band disturbances. For each level, according to the benchmark specifications, there are three tests. For each one, specifications have been set for the frequency and time domains. For the frequency domain, we evaluate: global attenuation (GA, in $\mathrm{dB})$, disturbance attenuation (DA, in $\mathrm{dB}$ ) and maximum amplification (MA) outside the attenuation frequencies (in pairs of $\mathrm{dB} @ \mathrm{~Hz}$ ). In the time domain, evaluation is done for: maximum value during the chirp (in $\mathrm{V}$ ) and transient performance. 
TABLE I

Simulation Results - Simple Step Test

\begin{tabular}{|c|c|c|c|c|c|c|c|}
\hline \multicolumn{8}{|c|}{ LEVEL 1} \\
\hline $\begin{array}{c}\text { Frequency } \\
(\mathbf{H z})\end{array}$ & $\begin{array}{l}\text { Global } \\
\text { (dB) }\end{array}$ & $\begin{array}{l}\text { Dist. Atten. } \\
\text { (dB) }\end{array}$ & $\begin{array}{l}\text { Max. Amp. } \\
(\text { dB @ Hz) }\end{array}$ & $\begin{array}{l}\text { Norm }^{2} \text { Trans. } \\
\quad\left(\times 10^{-3}\right)\end{array}$ & $\begin{array}{c}\text { Norm }^{2} \text { Res. } \\
\left(\times 10^{-3}\right)\end{array}$ & $\begin{array}{c}\text { Max. Val. } \\
\left(\times 10^{-3}\right)\end{array}$ & $\begin{array}{c}\text { TD } \\
\text { (ratio) }\end{array}$ \\
\hline 50 & 34.52 & 46.78 & $6.41 @ 59.38$ & 21.01 & 3.60 & 18.09 & 1.085 \\
\hline 55 & 34.47 & 50.04 & $4.21 @ 68.75$ & 24.14 & 3.65 & 20.70 & 1.081 \\
\hline 60 & 34.40 & 48.10 & $3.89 @ 68.75$ & 25.66 & 3.68 & 21.84 & 1.089 \\
\hline 65 & 34.40 & 48.52 & $3.59 @ 76.56$ & 18.87 & 3.74 & 19.36 & 1.073 \\
\hline 70 & 34.47 & 54.06 & $2.84 @ 79.69$ & 11.26 & 3.75 & 21.26 & 1.067 \\
\hline 75 & 34.84 & 54.26 & $3.06 @ 67.19$ & 7.84 & 3.68 & 22.82 & 1.067 \\
\hline 80 & 35.12 & 49.11 & $3.34 @ 67.19$ & 7.61 & 3.52 & 23.51 & 1.083 \\
\hline 85 & 34.88 & 49.97 & $3.40 @ 70.31$ & 8.69 & 3.50 & 23.55 & 1.091 \\
\hline 90 & 32.69 & 43.65 & $4.06 @ 78.13$ & 149.86 & 3.70 & 40.94 & 1.078 \\
\hline 95 & 23.78 & 40.36 & $7.86 @ 87.50$ & 200.93 & 4.60 & 38.80 & 1.073 \\
\hline \multicolumn{8}{|c|}{ LEVEL 2} \\
\hline $\begin{array}{l}\text { Frequency } \\
\quad(\mathbf{H z})\end{array}$ & $\begin{array}{c}\text { Global } \\
\text { (dB) }\end{array}$ & $\begin{array}{l}\text { Dist. Atten. } \\
\text { (dB)-(dB) }\end{array}$ & $\begin{array}{l}\text { Max. Amp. } \\
(\text { dB@Hz) }\end{array}$ & $\begin{array}{l}\text { Norm }^{2} \text { Trans. } \\
\quad\left(\times 10^{-3}\right)\end{array}$ & $\begin{array}{l}\text { Norm }^{2} \text { Res. } \\
\left(\times 10^{-3}\right)\end{array}$ & $\begin{array}{c}\text { Max. Val. } \\
\left(\times 10^{-3}\right)\end{array}$ & $\begin{array}{c}\text { TD } \\
\text { (ratio) }\end{array}$ \\
\hline $50-70$ & 39.68 & $45.48-50.32$ & $7.54 @ 59.38$ & 115.56 & 4.01 & 43.13 & 1.063 \\
\hline $55-75$ & 39.98 & $48.18-50.82$ & $6.29 @ 67.19$ & 261.46 & 3.94 & 51.75 & 1.066 \\
\hline $60-80$ & 40.51 & $45.91-46.35$ & $5.76 @ 68.75$ & 476.28 & 3.68 & 53.36 & 1.101 \\
\hline $65-85$ & 40.36 & $46.28-47.42$ & $5.65 @ 73.44$ & 375.31 & 3.71 & 49.41 & 1.090 \\
\hline $70-90$ & 38.99 & $52.14-43.25$ & $5.36 @ 76.56$ & 245.35 & 3.98 & 42.43 & 1.065 \\
\hline $75-95$ & 35.25 & $52.02-40.49$ & $8.50 @ 87.50$ & 144.05 & 4.68 & 49.25 & 10.110 \\
\hline \multicolumn{8}{|c|}{ LEVEL 3} \\
\hline $\begin{array}{l}\text { Frequency } \\
\quad(\mathbf{H z})\end{array}$ & $\begin{array}{c}\text { Global } \\
\text { (dB) }\end{array}$ & $\begin{array}{c}\text { Dist. Atten. } \\
\text { (dB)-(dB)-(dB) }\end{array}$ & $\begin{array}{l}\text { Max. Amp. } \\
(\text { dB@Hz) }\end{array}$ & $\begin{array}{l}\text { Norm }^{2} \text { Trans. } \\
\quad\left(\times 10^{-3}\right)\end{array}$ & $\begin{array}{l}\text { Norm }^{2} \text { Res. } \\
\left(\times 10^{-3}\right)\end{array}$ & $\begin{array}{l}\text { Max. Val. } \\
\left(\times 10^{-3}\right)\end{array}$ & $\begin{array}{c}\text { TD } \\
\text { (ratio) }\end{array}$ \\
\hline $50-65-80$ & 43.28 & $44.04-42.67-43.58$ & $7.52 @ 56.26$ & 29.80 & 3.99 & 50.43 & 1.068 \\
\hline $55-70-85$ & 43.26 & $46.34-47.80-45.53$ & $6.63 @ 62.50$ & 56.60 & 3.98 & 53.31 & 1.075 \\
\hline $60-75-90$ & 42.61 & $45.40-50.13-42.07$ & $6.78 @ 68.75$ & 131.40 & 4.07 & 65.38 & 1.075 \\
\hline $65-80-95$ & 40.48 & $45.29-42.19-38.66$ & $8.62 @ 87.50$ & 205.41 & 4.36 & 82.05 & 1.068 \\
\hline
\end{tabular}

The basic specification for transient performance is the requirement that the transient duration (TD) when a disturbance is applied, be smaller than $2 \mathrm{sec}$. Details of the measurement procedure can be found in [13]. From the point of view of the benchmark, this means that $2 \mathrm{sec}$ after application of a disturbance, the square of the truncated two norm has to be equal or smaller than 1.21 of the steady state value of the square of the truncated two norm of the residual force. The square of the truncated two norm is evaluated over an interval of $3 \mathrm{sec}$ both for transient and steady state, taking in account that disturbance is applied at $t=5 \mathrm{sec}$ and that steady state is evaluated between 17 and $20 \mathrm{sec}$. The square of the truncated two norm is denoted as $N 2 T(v: w)$ where $v$ and $w$ define the interval of computation. One defines:

$$
\begin{aligned}
\operatorname{TD}_{i} & =\frac{N 2 T(7: 10)}{N 2 T(17: 20)} \\
\Delta \text { Trans }_{i} & =T D_{i}-1.21, \text { if } T D_{i}>1.21 \\
\Delta \text { Trans }_{i} & =0, \text { if } T D_{i} \leq 1.21, \forall i=1, \ldots, M
\end{aligned}
$$

If $T D_{i}$ is smaller than 1.21 it means that the specifications for transient duration are satisfied (less or equal to $2 \mathrm{sec}$ ).

Other measurements are also considered in order to asses the performance of the approach. Such measurements include: quadratic norm of the transient and the residual (once the algorithm converges), the maximum value during the transient and the mean-square error during the chirp.

\section{A. Level 1}

For level one of the benchmark, the results, in the presence of a constant frequency disturbance (called Simple Step Test),
TABLE II

Simulation Results - LEVEl 1 - STEP FREQUENCY Changes Test

\begin{tabular}{|c|c|c|}
\hline \multicolumn{3}{|c|}{ SEQUENCE - 1 } \\
\hline $\begin{array}{c}\text { Frequency } \\
(\mathbf{H z})\end{array}$ & $\begin{array}{c}\text { Norm }{ }^{2} \text { Trans. } \\
\left(\times 10^{-3}\right)\end{array}$ & $\begin{array}{c}\text { Max. Val. } \\
\left(\times 10^{-3}\right)\end{array}$ \\
\hline $60 \rightarrow 70$ & 43.25 & 23.22 \\
\hline $70 \rightarrow 60$ & 40.17 & 21.01 \\
\hline $60 \rightarrow 50$ & 31.51 & 18.76 \\
\hline $50 \rightarrow 60$ & 50.93 & 30.19 \\
\hline \multicolumn{3}{|c|}{ SEQUENCE - 2 } \\
\hline $\begin{array}{c}\text { Frequency } \\
(\text { Hz) })\end{array}$ & $\begin{array}{c}\text { Norm }{ }^{2} \text { Trans. } \\
\left(\times 10^{-3}\right)\end{array}$ & $\begin{array}{c}\text { Max. Val. } \\
\left(\times 10^{-3}\right)\end{array}$ \\
\hline $75 \rightarrow 85$ & 44.71 & 21.24 \\
\hline $85 \rightarrow 75$ & 52.24 & 21.29 \\
\hline $75 \rightarrow 65$ & 45.09 & 19.87 \\
\hline $65 \rightarrow 75$ & 43.27 & 22.94 \\
\hline \multicolumn{3}{|c|}{ SEQUENCE - 3 } \\
\hline $\begin{array}{c}\text { Frequency } \\
(\text { Nz) })\end{array}$ & $\begin{array}{c}\text { Norm }{ }^{2} \text { Trans. } \\
\left(\times 10^{-3}\right)\end{array}$ & $\begin{array}{c}\text { Max. Val. } \\
\left(\times 10^{-3}\right)\end{array}$ \\
\hline $85 \rightarrow 95$ & 56.03 & 23.15 \\
\hline $95 \rightarrow 85$ & 104.22 & 36.57 \\
\hline $85 \rightarrow 75$ & 51.71 & 22.15 \\
\hline $75 \rightarrow 85$ & 44.10 & 21.12 \\
\hline \multicolumn{3}{|c|}{} \\
\hline
\end{tabular}

are summarised in Table I. As it can be seen, in general, all the specifications were fulfilled (one notices however a global attenuation below the required value of $24 \mathrm{~dB}$ at $95 \mathrm{~Hz}$ ). Since this approach uses the frequency estimation described in [22], [21], the performance of the control scheme relies on how fast the frequency is estimated, thereby the transient duration results are strongly linked to this issue. For the case when step changes in the frequency of the disturbance occur (called Step Frequency Changes Test), the results are shown in Table II. The results for the chirp disturbance test (called 
Chirp Test) are summarized in Table III. $\nearrow$ denotes linearly increasing frequency chirp disturbance, while $\searrow$ is used to denote the disturbance with linearly decreasing frequencies. The maximum value during the chirp periods did not exceed the imposed limit of $0.1 \mathrm{~V}$.

TABLE III

Simulation Results - LeVEl 1 - ChiRP Test

\begin{tabular}{|c|c|c|}
\hline & Error-Mean Square Value & Error-Maximum Value \\
\hline$\nearrow$ & $39.229 \times 10^{-6}$ & $13.228 \times 10^{-3}$ \\
\hline$\searrow$ & $53.673 \times 10^{-6}$ & $18.688 \times 10^{-3}$ \\
\hline
\end{tabular}

TABLE IV

Simulation Results - LeVel 2 - SteP FReQuenCy CHANGES TEST

\begin{tabular}{|c|c|c|}
\hline \multicolumn{3}{|c|}{ SEQUENCE - 1 } \\
\hline $\begin{array}{c}\text { Frequency } \\
(\mathbf{H z})\end{array}$ & $\begin{array}{c}\text { Norm }^{2} \text { Trans. } \\
\left(\times 10^{-3}\right)\end{array}$ & $\begin{array}{c}\text { Max. Val. } \\
\left(\times 10^{-3}\right)\end{array}$ \\
\hline$[55,75] \rightarrow[60,80]$ & 37.65 & 34.75 \\
\hline$[60,80] \rightarrow[55,75]$ & 36.85 & 33.37 \\
\hline$[55,75] \rightarrow[50,70]$ & 38.40 & 32.51 \\
\hline$[50,70] \rightarrow[55,75]$ & 43.66 & 36.21 \\
\hline \multicolumn{3}{|c|}{ SEQUENCE - 2 } \\
\hline $\begin{array}{c}\text { Frequency } \\
(\mathbf{H z})\end{array}$ & $\begin{array}{c}\text { Norm }^{2} \text { Trans. } \\
\left(\times 10^{-3}\right)\end{array}$ & $\begin{array}{c}\text { Max. Val. } \\
\left(\times 10^{-3}\right)\end{array}$ \\
\hline$[70,90] \rightarrow[75,95]$ & 40.40 & 28.90 \\
\hline$[75,95] \rightarrow[70,90]$ & 64.08 & 40.62 \\
\hline$[70,90] \rightarrow[65,85]$ & 38.14 & 31.23 \\
\hline$[65,85] \rightarrow[70,90]$ & 37.38 & 31.43 \\
\hline
\end{tabular}

\section{B. Level 2}

For the second level of the benchmark, the results of the proposed approach during the Simple Step Test are shown in Table I. Almost all the benchmark specifications were satisfied, the only criteria which is not met being the maximum amplification and the transient duration ratio at $75-95 \mathrm{~Hz}$ The other values for the TD ratio are within the requirements for the benchmark. For Step Frequency Changes Test and Chirp Test, the limits were respected as it can be seen in Tables IV and V.

TABLE V

Simulation Results - LEVEl 2 - ChiRp TEST

\begin{tabular}{|c|c|c|}
\hline & Error-Mean Square Value & Error-Maximum Value \\
\hline$\nearrow$ & $25.998 \times 10^{-6}$ & $13.416 \times 10^{-3}$ \\
\hline$\searrow$ & $29.678 \times 10^{-6}$ & $16.466 \times 10^{-3}$ \\
\hline
\end{tabular}

TABLE VI

Simulation Results - LeVEl 3 - Step FReQuency CHANGES TEST

\begin{tabular}{|c|c|c|}
\hline \multicolumn{3}{|c|}{ SEQUENCE - 1 } \\
\hline $\begin{array}{c}\text { Frequency } \\
(\mathbf{H z})\end{array}$ & $\begin{array}{c}\text { Norm }^{2} \text { Trans. } \\
\left(\times 10^{-3}\right)\end{array}$ & $\begin{array}{c}\text { Max. Val. } \\
\left(\times 10^{-3}\right)\end{array}$ \\
\hline$[55,70,85] \rightarrow[60,75,90]$ & 84.74 & 58.41 \\
\hline$[60,75,90] \rightarrow[55,70,85]$ & 86.05 & 59.28 \\
\hline$[55,70,85] \rightarrow[50,65,80]$ & 90.77 & 58.00 \\
\hline$[50,65,80] \rightarrow[55,70,85]$ & 91.86 & 63.18 \\
\hline \multicolumn{3}{|c|}{ SEQUENCE - 2 } \\
\hline$[60,75,90] \rightarrow[65,80,95]$ & 85.96 & 53.62 \\
\hline$[65,80,95] \rightarrow[60,75,90]$ & 113.25 & 62.22 \\
\hline$[60,75,90] \rightarrow[55,70,85]$ & 85.49 & 60.84 \\
\hline$[55,70,85] \rightarrow[60,75,90]$ & 84.16 & 58.74 \\
\hline
\end{tabular}

\section{Level 3}

On the third level, for Simple Step Test, only at $95 \mathrm{~Hz}$ the disturbance attenuation requirement was not achieved as imposed by the benchmark specifications (see Table I). The Step Frequency Changes and Chirp Tests results are shown in Tables VI and VII.

TABLE VII

Simulation Results - LEVEL 3 - ChiRp TEST

\begin{tabular}{|c|c|c|}
\hline & Error-Mean Square Value & Error-Maximum Value \\
\hline$\nearrow$ & $15.830 \times 10^{-6}$ & $12.494 \times 10^{-3}$ \\
\hline$\searrow$ & $17.190 \times 10^{-6}$ & $12.783 \times 10^{-3}$ \\
\hline
\end{tabular}

\section{EXPERIMENTAL RESULTS}

Using the same central controller and frequency estimation configuration (Section V), the following real-time results were obtained.

TABLE IX

EXPERIMENTAL RESUlTS - LEVEL 1 - STEP FREQUENCY CHANGes Test

\begin{tabular}{|c|c|c|}
\hline \multicolumn{3}{|c|}{ SEQUENCE - 1} \\
\hline $\begin{array}{c}\text { Frequency } \\
(\mathrm{Hz})\end{array}$ & $\begin{array}{c}\text { Norm }^{2} \text { Trans. } \\
\left(\times 10^{-3}\right)\end{array}$ & $\begin{array}{c}\text { Max. Val. } \\
\left(\times 10^{-3}\right)\end{array}$ \\
\hline $60 \rightarrow 70$ & 49.83 & 24.68 \\
\hline $70 \rightarrow 60$ & 50.84 & 24.68 \\
\hline $60 \rightarrow 50$ & 63.82 & 23.46 \\
\hline $50 \rightarrow 60$ & 81.38 & 39.39 \\
\hline \multicolumn{3}{|c|}{ SEQUENCE - 2} \\
\hline $\begin{array}{c}\text { Frequency } \\
\text { (Hz) }\end{array}$ & $\begin{array}{c}\text { Norm }^{2} \text { Trans. } \\
\left(\times 10^{-3}\right)\end{array}$ & $\begin{array}{c}\text { Max. Val. } \\
\left(\times 10^{-3}\right)\end{array}$ \\
\hline $75 \rightarrow 85$ & 47.40 & 20.99 \\
\hline $85 \rightarrow 75$ & 52.77 & 20.993 \\
\hline $75 \rightarrow 65$ & 52.55 & 23.44 \\
\hline $65 \rightarrow 75$ & 48.34 & 24.66 \\
\hline \multicolumn{3}{|c|}{ SEQUENCE - 3} \\
\hline $\begin{array}{c}\text { Frequency } \\
(\mathrm{Hz})\end{array}$ & $\begin{array}{c}\text { Norm }^{2} \text { Trans. } \\
\quad\left(\times 10^{-3}\right)\end{array}$ & $\begin{array}{c}\text { Max. Val. } \\
\left(\times 10^{-3}\right)\end{array}$ \\
\hline $85 \rightarrow 95$ & 59.21 & 15.79 \\
\hline $95 \rightarrow 85$ & 95.33 & 32.01 \\
\hline $85 \rightarrow 75$ & 52.48 & 19.75 \\
\hline $75 \rightarrow 85$ & 46.39 & 19.75 \\
\hline
\end{tabular}

\section{A. Level 1 Results}

In Table VIII, the experimental results for Simple Step Level 1 are summarised. The most important differences between these results and the ones obtained in simulation concern the maximum amplification (this is due probably to the model uncertainties, some small error in the estimation of the frequency of the disturbances, and also to the measurement noise present in the real time evaluations which is not the same as the one used in simulations). Except that, the results are close to those obtained in simulation.

Table IX shows the Step Frequency Changes Test results for this level. In most of the cases, one obtains faster transients in real-time than in simulation. Also for the Chirp Test, the results are generally better than in simulation (Table X).

The time responses of the system in open loop and in closed loop with the proposed disturbance attenuation approach are shown in Fig. 4 for specific disturbance characteristics (see figure for details). A sufficient level of attenuation is observed in all of the tests. In the case of the simple step test, the 
TABLE VIII

EXPERIMENTAL RESUltS - Simple Step TEST

\begin{tabular}{|c|c|c|c|c|c|c|c|}
\hline \multicolumn{8}{|c|}{ LEVEL 1} \\
\hline $\begin{array}{l}\text { Frequency } \\
(\mathbf{H z})\end{array}$ & $\begin{array}{l}\text { Global } \\
\text { (dB) }\end{array}$ & $\begin{array}{l}\text { Dist. Atten. } \\
\text { (dB) }\end{array}$ & $\begin{array}{c}\text { Max. Amp. } \\
(\mathrm{dB} @ \mathbf{H z})\end{array}$ & $\begin{array}{c}\text { Norm }^{2} \text { Trans. } \\
\left(\times 10^{-3}\right)\end{array}$ & $\begin{array}{c}\text { Norm }^{2} \text { Res. } \\
\left(\times 10^{-3}\right)\end{array}$ & $\begin{array}{l}\text { Max. Val. } \\
\left(\times 10^{-3}\right)\end{array}$ & $\begin{array}{c}\text { TD } \\
\text { (ratio) }\end{array}$ \\
\hline 50 & 36.17 & 37.54 & $11.61 @ 65.63$ & 62.06 & 6.61 & 23.57 & 1.013 \\
\hline 55 & 38.50 & 49.35 & 8.15@120.31 & 33.31 & 3.60 & 21.81 & 1.310 \\
\hline 60 & 36.74 & 49.20 & $9.53 @ 46.88$ & 26.13 & 4.11 & 20.59 & 1.088 \\
\hline 65 & 36.25 & 48.45 & 8.03@134.38 & 15.17 & 3.56 & 22.35 & 0.875 \\
\hline 70 & 34.23 & 52.76 & $10.66 @ 134.38$ & 9.88 & 3.67 & 19.37 & 1.097 \\
\hline 75 & 33.31 & 46.91 & $5.99 @ 134.38$ & 8.45 & 3.64 & 18.62 & 1.029 \\
\hline 80 & 32.65 & 48.32 & $7.48 @ 276.56$ & 16.05 & 3.65 & 20.56 & 0.985 \\
\hline 85 & 32.58 & 48.07 & 12.03@73.44 & 20.85 & 3.55 & 22.33 & 1.120 \\
\hline 90 & 31.19 & 47.38 & $8.49 @ 14.06$ & 21.18 & 3.64 & 24.79 & 1.071 \\
\hline 95 & 28.83 & 38.52 & $10.85 @ 82.21$ & 21.32 & 3.71 & 27.22 & 1.094 \\
\hline \multicolumn{8}{|c|}{ LEVEL 2} \\
\hline $\begin{array}{l}\text { Frequency } \\
(\mathbf{H z})\end{array}$ & $\begin{array}{c}\text { Global } \\
\text { (dB) }\end{array}$ & $\begin{array}{l}\text { Dist. Atten. } \\
\text { (dB)-(dB) }\end{array}$ & $\begin{array}{l}\text { Max. Amp. } \\
(\mathrm{dB} @ \mathbf{H z})\end{array}$ & $\begin{array}{l}\text { Norm }^{2} \text { Trans. } \\
\quad\left(\times 10^{-3}\right)\end{array}$ & $\begin{array}{l}\text { Norm }^{2} \text { Res. } \\
\left(\times 10^{-3}\right)\end{array}$ & $\begin{array}{l}\text { Max. Val. } \\
\left(\times 10^{-3}\right)\end{array}$ & $\begin{array}{c}\text { TD } \\
\text { (ratio) }\end{array}$ \\
\hline 50-70 & 38.44 & $39.73-47.27$ & $9.29 @ 56.25$ & 50.36 & 7.68 & 34.56 & 0.983 \\
\hline $55-75$ & 40.22 & $48.89-40.72$ & $9.05 @ 270.31$ & 435.20 & 4.74 & 63.44 & 0.982 \\
\hline $60-80$ & 39.07 & $49.94-47.17$ & $9.96 @ 68.75$ & 51.69 & 3.54 & 35.96 & 0.939 \\
\hline $65-85$ & 35.86 & $43.67-43.89$ & $9.75 @ 104.69$ & 25.08 & 5.13 & 48.52 & 0.939 \\
\hline $70-90$ & 35.44 & $47.23-39.01$ & 8.70@134.38 & 230.78 & 4.22 & 84.22 & 0.923 \\
\hline $75-95$ & 35.44 & $47.23-39.01$ & $8.40 @ 81.25$ & 131.21 & 4.02 & 37.69 & 1.019 \\
\hline \multicolumn{8}{|c|}{ LEVEL 3} \\
\hline $\begin{array}{l}\text { Frequency } \\
\quad(\mathbf{H z})\end{array}$ & $\begin{array}{c}\text { Global } \\
\text { (dB) }\end{array}$ & $\begin{array}{c}\text { Dist. Atten. } \\
\text { (dB)-(dB)-(dB) }\end{array}$ & $\begin{array}{l}\text { Max. Amp. } \\
(\mathrm{dB} @ \mathrm{~Hz})\end{array}$ & $\begin{array}{l}\text { Norm }^{2} \text { Trans. } \\
\quad\left(\times 10^{-3}\right)\end{array}$ & $\begin{array}{l}\text { Norm }^{2} \text { Res. } \\
\left(\times 10^{-3}\right)\end{array}$ & $\begin{array}{l}\text { Max. Val. } \\
\left(\times 10^{-3}\right)\end{array}$ & $\begin{array}{c}\text { TD } \\
\text { (ratio) }\end{array}$ \\
\hline $50-65-80$ & 41.87 & $32.47-34.95-43.68$ & $8.02 @ 71.69$ & 44.00 & 6.46 & 45.72 & 1.052 \\
\hline $55-70-85$ & 42.53 & $46.70-46.16-48.35$ & $10.99 @ 62.50$ & 92.26 & 4.93 & 58.58 & 0.890 \\
\hline $60-75-90$ & 40.77 & $49.59-42.09-42.37$ & $8.70 @ 67.19$ & 173.46 & 5.40 & 64.68 & 0.972 \\
\hline $65-80-95$ & 41.43 & $44.85-43.74-36.49$ & $9.88 @ 87.50$ & 340.05 & 4.47 & 57.37 & 0.973 \\
\hline
\end{tabular}

power spectral density (PSD) estimate of the open loop and the effective attenuation are shown in Fig. 5. The tests shown in these figure have been selected to be the same for all the participants in order to help evaluate the various approaches.

TABLE X

EXPERIMENTAL RESUlTS - LEVEL 1 - CHIRP TEST

\begin{tabular}{|c|c|c|}
\hline & Error-Mean Square Value & Error-Maximum Value \\
\hline$\nearrow$ & $31.783 \times 10^{-6}$ & $16.730 \times 10^{-3}$ \\
\hline$\searrow$ & $35.878 \times 10^{-6}$ & $11.828 \times 10^{-3}$ \\
\hline
\end{tabular}
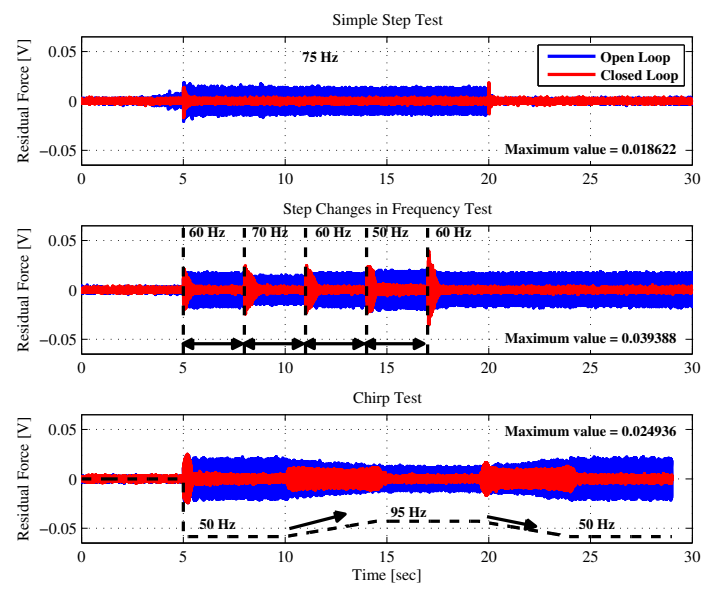

Fig. 4. Time response results for Level 1 - experimental.

\section{B. Level 2 Results}

Simple Step Test results for this level are shown in Table VIII and it can be noticed that the algorithm provides in general good results within the specifications of the benchmark

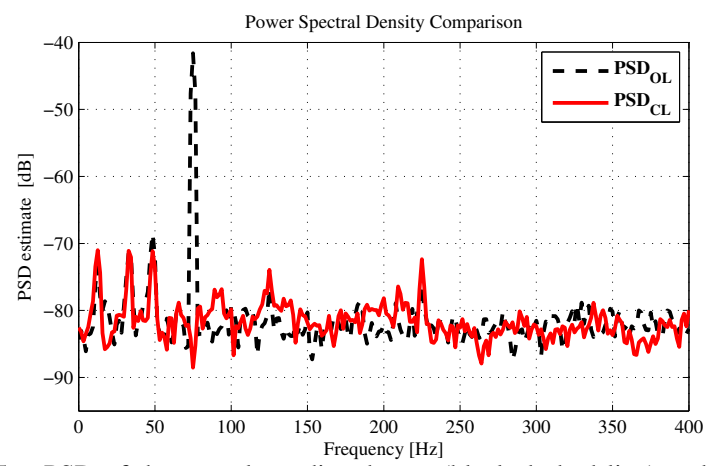

Fig. 5. PSD of the open loop disturbance (black dashed line) and effective attenuation (red line) for $75 \mathrm{~Hz}$ - experimental. TABLE XI

EXPERIMENTAL RESUlTS - LEVEL 2 - STEP FREQUENCY CHANGES TEST

\begin{tabular}{|c|c|c|}
\hline \multicolumn{3}{|c|}{ SEQUENCE - 1} \\
\hline $\begin{array}{c}\text { Frequency } \\
(\mathbf{H z})\end{array}$ & $\begin{array}{l}\text { Norm }^{2} \text { Trans. } \\
\left(\times 10^{-3}\right)\end{array}$ & $\begin{array}{c}\text { Max. Val. } \\
\left(\times 10^{-3}\right)\end{array}$ \\
\hline$[55,75] \rightarrow[60,80]$ & 46.39 & 19.75 \\
\hline$[60,80] \rightarrow[55,75]$ & 33.71 & 37.39 \\
\hline$[55,75] \rightarrow[50,70]$ & 50.66 & 36.70 \\
\hline$[50,70] \rightarrow[55,75]$ & 45.62 & 41.61 \\
\hline \multicolumn{3}{|c|}{ SEQUENCE - 2} \\
\hline $\begin{array}{l}\text { Frequency } \\
(\mathbf{H z})\end{array}$ & $\begin{array}{l}\text { Norm }^{2} \text { Trans. } \\
\quad\left(\times 10^{-3}\right)\end{array}$ & $\begin{array}{c}\text { Max. Val. } \\
\left(\times 10^{-3}\right)\end{array}$ \\
\hline$[70,90] \rightarrow[75,95]$ & 44.15 & 35.49 \\
\hline$[75,95] \rightarrow[70,90]$ & 54.60 & 36.82 \\
\hline$[70,90] \rightarrow[65,85]$ & 38.19 & 34.26 \\
\hline$[65,85] \rightarrow[70,90]$ & 41.22 & 34.37 \\
\hline
\end{tabular}

with the exception of the maximum amplification which is over the limit. Tables XI and XII show the results for the Step Frequency Changes and the Chirp Test.

The time responses of the system in open loop and in closed loop with the proposed disturbance attenuation approach are 
shown in Fig. 6 for specific disturbance characteristics. A sufficient level of attenuation is observed in all of the tests. In the case of the simple step test, the power spectral density (PSD) estimate of the open loop and the effective attenuation are shown in Fig. 7.
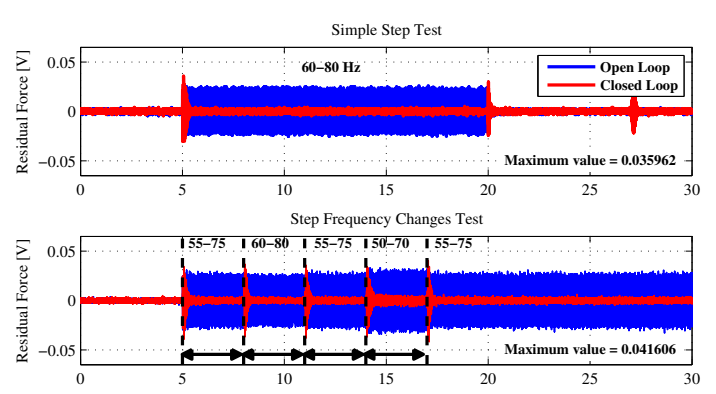

Chirp Test

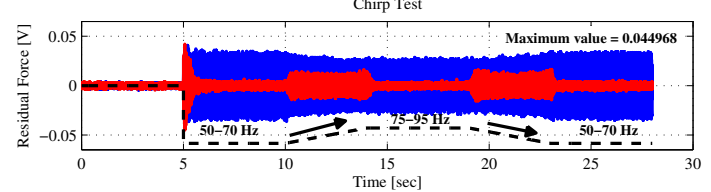

Fig. 6. Time response results for Level 2 - experimental.

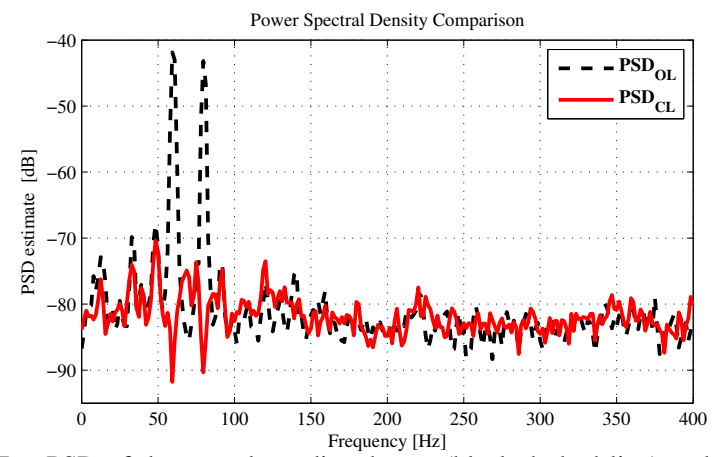

Fig. 7. PSD of the open loop disturbance (black dashed line) and effective attenuation (red line) for $60-80 \mathrm{~Hz}$ - experimental.

TABLE XII

EXPERIMENTAL RESUlTS - LEVEL 2 - CHIRP TEST

\begin{tabular}{|c|c|c|}
\hline & Error-Mean Square Value & Error-Maximum Value \\
\hline$\nearrow$ & $34.007 \times 10^{-6}$ & $16.307 \times 10^{-3}$ \\
\hline$\searrow$ & $35.600 \times 10^{-6}$ & $16.307 \times 10^{-3}$ \\
\hline
\end{tabular}

TABLE XIII

EXPERIMENTAL RESULTS - LEVEL 3 - STEP FREQUENCY CHANGES TEST

\begin{tabular}{|c|c|c|}
\hline \multicolumn{3}{|c|}{ SEQUENCE - 1 } \\
\hline $\begin{array}{c}\text { Frequency } \\
(\mathbf{H z})\end{array}$ & $\begin{array}{c}\text { Norm }^{2} \text { Trans. } \\
\left(\times 10^{-3}\right)\end{array}$ & $\begin{array}{c}\text { Max. Val. } \\
\left(\times 10^{-3}\right)\end{array}$ \\
\hline$[55,70,85] \rightarrow[60,75,90]$ & 97.33 & 65.06 \\
\hline$[60,75,90] \rightarrow[55,70,85]$ & 86.38 & 64.84 \\
\hline$[55,70,85] \rightarrow[50,65,80]$ & 103.87 & 62.61 \\
\hline$[50,65,80] \rightarrow[55,70,85]$ & 115.15 & 68.74 \\
\hline \multicolumn{3}{|c|}{ SEQUENCE - 2 } \\
\hline$[60,75,90] \rightarrow[65,80,95]$ & 96.45 & 57.76 \\
\hline$[65,80,95] \rightarrow[60,75,90]$ & 100.68 & 61.44 \\
\hline$[60,75,90] \rightarrow[55,70,85]$ & 85.00 & 61.44 \\
\hline$[55,70,85] \rightarrow[60,75,90]$ & 96.50 & 63.57 \\
\hline
\end{tabular}

\section{Level 3 Results}

In the most challenging level, the good performance of the algorithm is proved since the benchmark specifications are passed for all the objectives in the Simple Step Test (Table VIII). Table XIII shows the results for the Step Frequency Changes Test. It can be observed that both the two norm transient and the maximum value are worse than for the previous level but the values are still acceptable from the benchmark specifications point of view. Meanwhile, in Table XIV the Chirp Test evaluation is shown and the result is even better than for the previous level.

The time responses of the system in open loop and in closed loop with the proposed disturbance attenuation approach are shown in Fig. 8 for specific disturbance characteristics. A sufficient level of attenuation is observed in all of the tests. In the case of the simple step test, the power spectral density (PSD) estimate of the open loop and the effective attenuation are shown in Fig. 9.
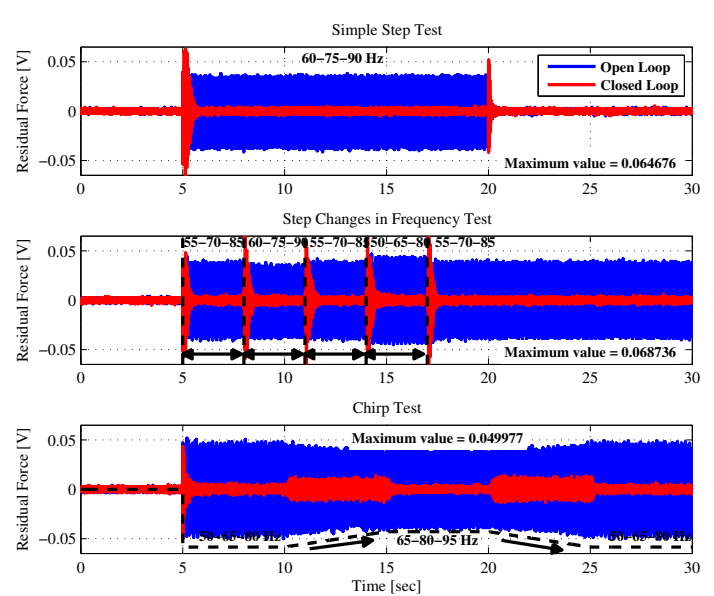

Fig. 8. Time response results for Level 3 - experimental.

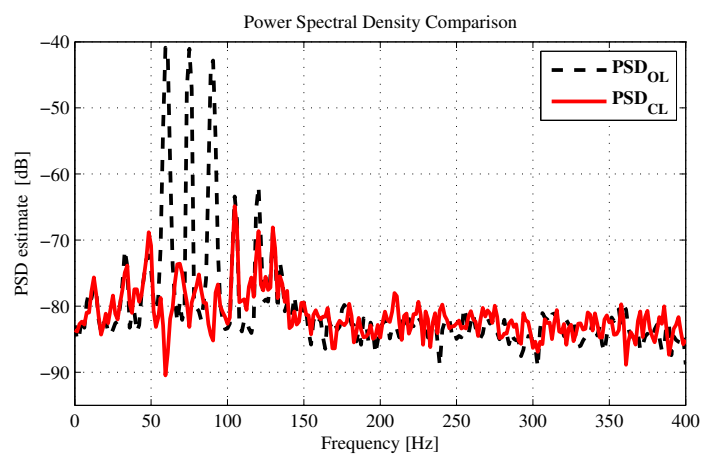

Fig. 9. PSD of the open loop disturbance (black dashed line) and effective attenuation (red line) for $60-75-90 \mathrm{~Hz}$ - experimental.

TABLE XIV

EXPERIMENTAL RESUlTS - LEVEL 3 - CHIRP TEST

\begin{tabular}{|c|c|c|}
\hline & Error-Mean Square Value & Error-Maximum Value \\
\hline$\nearrow$ & $19.874 \times 10^{-6}$ & $13.749 \times 10^{-3}$ \\
\hline$\searrow$ & $20.479 \times 10^{-6}$ & $13.749 \times 10^{-3}$ \\
\hline
\end{tabular}

\section{Comparison with simulation results}

The comparison of the GA, DA, MA and TD between simulation (Table I) and experimental (Table VIII) results for the Single Step Test is shown in Fig. 10 for Level 1 of the benchmark, in Fig. 11 for Level 2, and in Fig. 12 for Level 3. The interpretation of these results is given next. For Level 
1, one observes in Fig. 10 very similar results with respect to global attenuation and disturbance attenuation. However, for the MA the results obtained experimentally are worse than those obtained in simulation and the main reason for this is that the measurement noise used in simulations is not representative of the one on the real time system (see also [17]) but also to possible model uncertainties. Nevertheless, a modified design of the central controller, which decreases even more the input sensitivity function's amplitude above $100 \mathrm{~Hz}$ (by introduction of 4 additional BSFs in the input sensitivity function). Such a central controller has improved the performances for the Level 1 but not for Levels 2 and 3.

With respect to the TD ratio (i.e., ratio between the squared two norm of the residual force from 7 to 10 seconds and the squared two norm of the residual force from 17 to 20 seconds, as described by eqs. (41)-(43)), the experimental results seem overall better than those obtained in simulation (especially for Levels 2, Fig. 11, and 3, Fig. 12).

Another aspect is the maximum amplification, which, by analysing the Level 3 comparison in Fig. 12, is significantly improved with respect to the previous two levels and very close to the simulation results with the single exception of the rejection of a disturbance composed of sinusoids at 55, 70, and $85 \mathrm{~Hz}$.

To complete the comparison, particular disturbance configurations are chosen and further analysed. In Fig. 13, the PSD estimates of the effective attenuation/amplification of the residual during the Simple Step Test, are shown ${ }^{5}$. It can be seen that measurement noise is present in the experimental results but the level of the noise in closed loop remains below the benchmark specifications (i.e., below the accepted MA).For the Step Frequency Changes Test, Fig. 14 shows very close results between simulation and real time and a satisfactory level of attenuation for both. The frequency estimation comparison is shown in Fig. 15 for the same protocol as in Fig. 14. It is notable that transient durations inFig. 14 are related to those in Fig. 15. Finally, a Chirp Test comparison is provided in Fig. 16 and allows to conclude that both in simulation and in real time the proposed approach gives satisfactory results. Robustness is also shown by the similarity of the simulation and real time results.

\section{CONCLUDING REMARKS}

The idea of designing an adaptive controller on which the value of the attenuation can be imposed, proved to be efficient in practice. A single central controller has been used for the three levels of the benchmark in both simulation and in real time.

Experimental results on the benchmark platform have shown a reasonable good coherence between simulation and real time results. The IBSF simplifies the design, since it allows to obtain good results without the redesign of the central controller for each benchmark level, and seems to be less sensitive with respect to controller design, plant model uncertainties at various frequencies, and to measurement noise. In

\footnotetext{
${ }^{5}$ Note that the two PSDs are computed with 512 points windows and do not allow to view the attenuations of $80 \mathrm{~dB}$ introduced by the BSFs.
}

GA

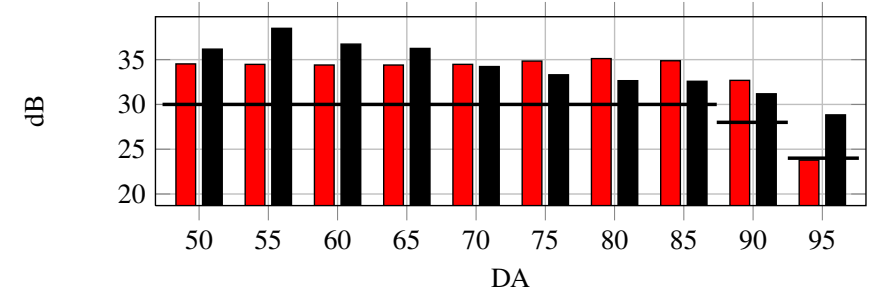

$\cong$

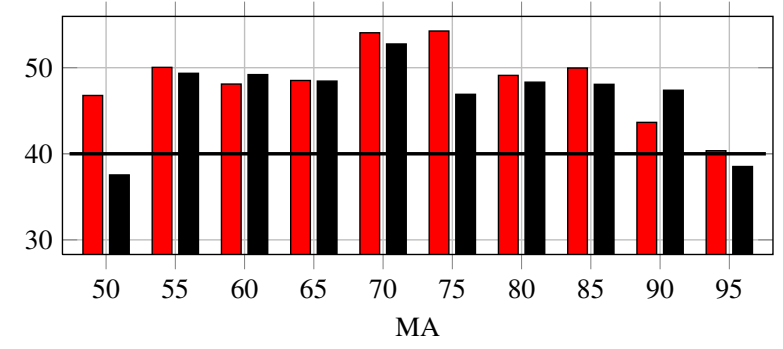

$\vartheta$
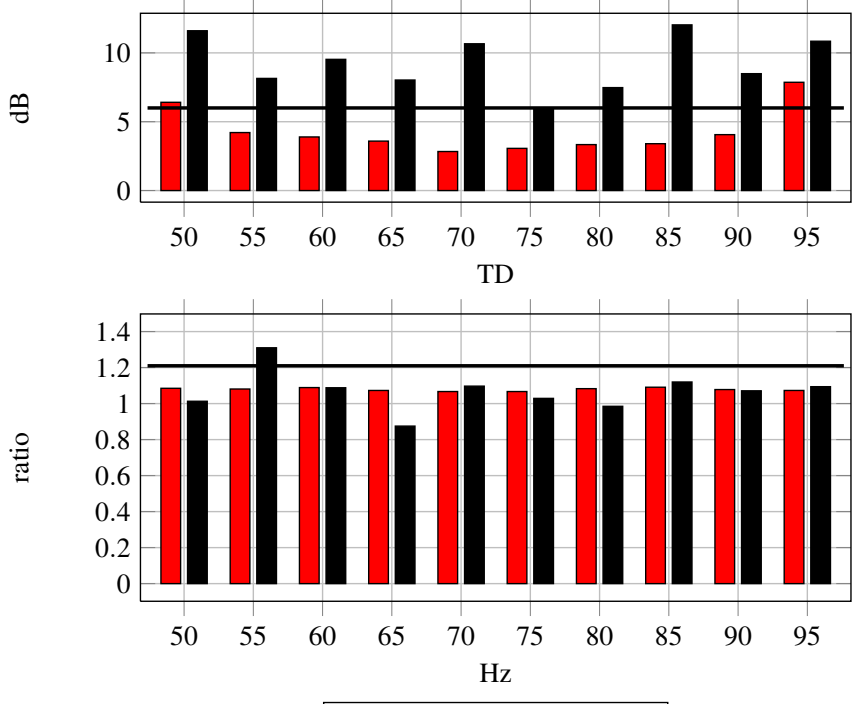

TuSimulation I Real-time

Fig. 10. Level 1 comparison between simulation and experimental results.

terms of computational complexity, indirect adaptive control approaches are in general more demanding than direct adaptive control approaches ([14]), but the compromise between robust performance and computational complexity makes it appealing for the rejection of multiple narrow-band disturbances.

Among the advantages of this approach we should mention an easy and systematic controller design assuring simultaneously a good profile for the sensitivity functions in order to guarantee a good robustness with respect to plant model uncertainties and low noise amplification outside the region of attenuation.

\section{REFERENCES}

[1] T.-B. Airimitoaie and I.D. Landau. Indirect adaptive attenuation of multiple narrow-band disturbances applied to active vibration control. Control Systems Technology, IEEE Transactions on, 2013. accepted.

[2] F. Ben Amara, P.T. Kabamba, and A.G. Ulsoy. Adaptive sinusoidal disturbance rejection in linear discrete-time systems - Parts I and II. Journal of Dynamic Systems Measurement and Control, 121:648-659, 1999. 
GA

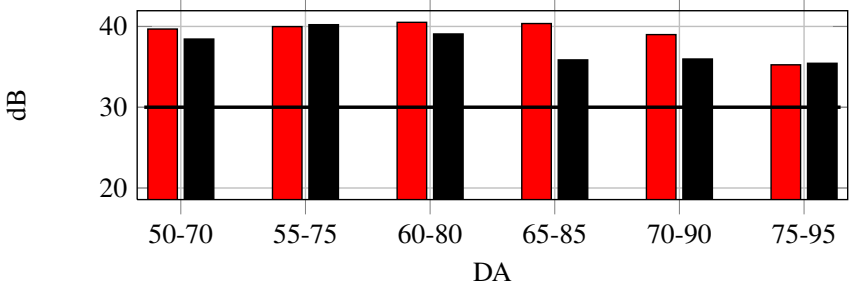

$\cong$

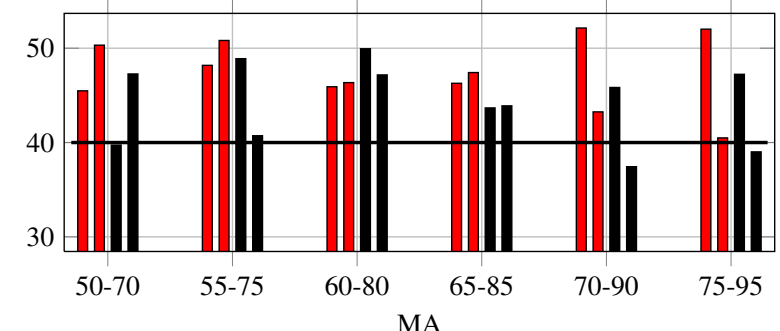

$\approx$
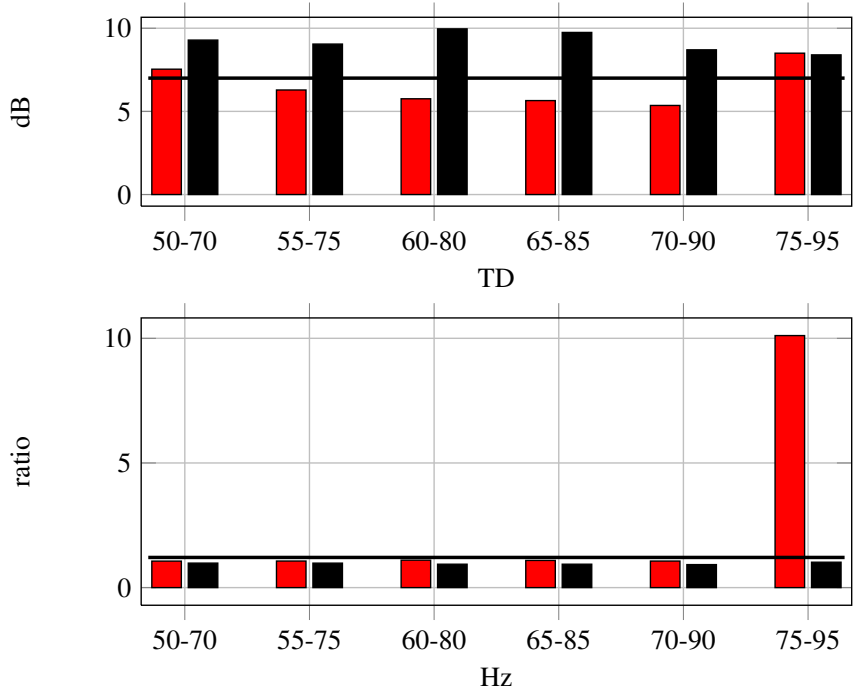

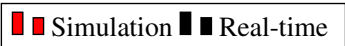

Fig. 11. Level 2 comparison between simulation and experimental results.

[3] B.D.O. Anderson. From Youla-Kucera to identification, adaptive and nonlinear control. Automatica, 34:1485-1506, 1998.

[4] Alexey A. Bobtsov, Denis Efimov, Anton A. Pyrkin, and Ali Zolghadri. Switched algorithm for frequency estimation with noise rejection. $\mathrm{Au}$ tomatic Control, IEEE Transactions on, 57(9):2400-2404, 2012.

[5] Alexey A. Bobtsov and Anton A. Pyrkin. Cancelation of unknown multiharmonic disturbance for nonlinear plant with input delay. International Journal of Adaptive Control and Signal Processing, 26(4):302315,2012

[6] M. Bodson. Rejection of periodic distrubances of unknown and timevarying frequency. Int. J. of Adapt. Contr. and Sign. Proc., 19:67-88, 2005.

[7] M. Bodson and S.C. Douglas. Adaptive algorithms for the rejection of sinusosidal disturbances with unknown frequency. Automatica, 33:22132221, 1997.

[8] Bor-Sen Chen, Tsang-Yi Yang, and Bin-Hong Lin. Adaptive notch filter by direct frequency estimation. Signal Processing, 27(2):161 - 176, 1992.

[9] $\mathrm{Xu}$ Chen and M. Tomizuka. A minimum parameter adaptive approach for rejecting multiple narrow-band disturbances with application to hard disk drives. Control Systems Technology, IEEE Transactions on 20(2):408 -415, march 2012.

[10] Raymond A. de Callafon and Charles E. Kinney. Robust estimation and adaptive controller tuning for variance minimization in servo systems. Journal of Advanced Mechanical Design, Systems, and Manufacturing,
GA

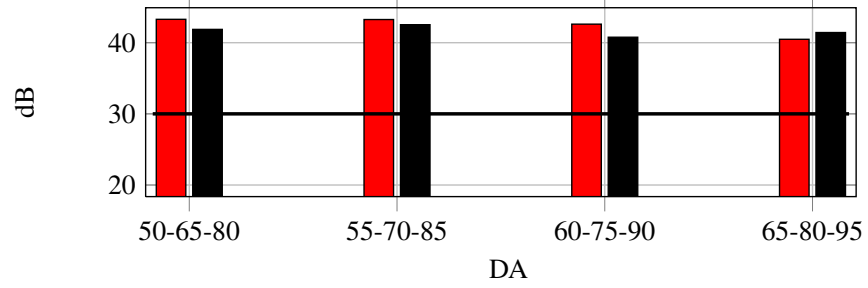

$\because$

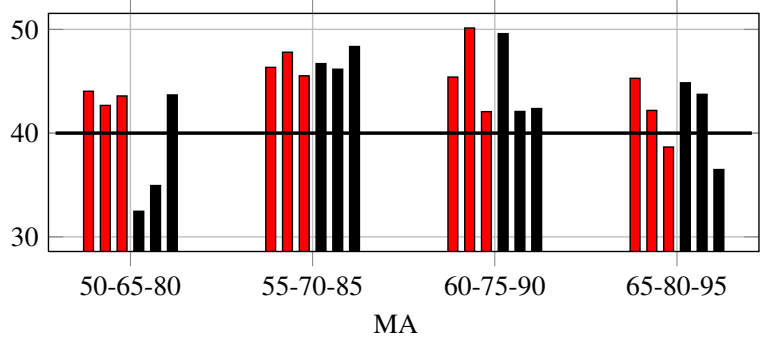

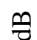
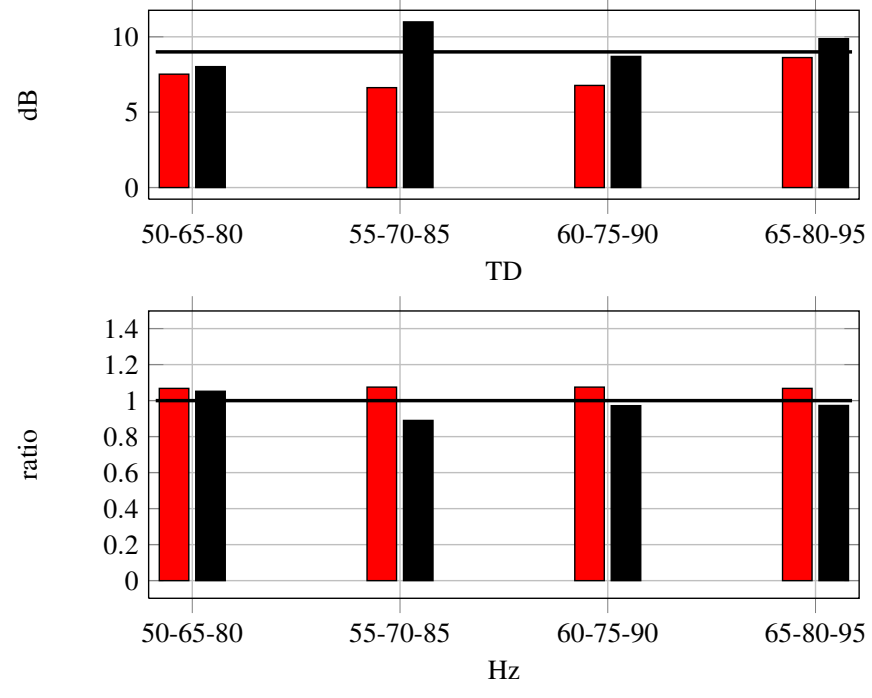

एuSimulation $\square$ Real-time

Fig. 12. Level 3 comparison between simulation and experimental results.

4(1): $130-142,2010$.

[11] B.A. Francis and W.M. Wonham. The internal model principle of control theory. Automatica, 12(5):457 - 465, 1976.

[12] Liu Hsu, Romeo Ortega, and Gilney Damm. A globally convergent frequency estimator. IEEE Trans. Autom. Control, 4(4):698-713, 1999.

[13] I.D. Landau, T.-B. Airimiţoaie, A. Castellanos Silva, and G. Buche Benchmark on adaptive regulation. http://www.gipsa-lab.grenoble-inp. fr/ ioandore.landau/benchmark_adaptive_regulation/.

[14] I.D. Landau, M. Alma, J.J. Martinez, and G. Buche. Adaptive suppression of multiple time-varying unknown vibrations using an inertial actuator. Control Systems Technology, IEEE Transactions on, 19(6):1327 -1338 , nov. 2011.

[15] I.D. Landau, A. Constantinescu, and D. Rey. Adaptive narrow band disturbance rejection applied to an active suspension - an internal model principle approach. Automatica, 41(4):563-574, 2005.

[16] I.D. Landau and G. Zito. Digital Control Systems - Design, Identification and Implementation. Springer, London, 2005.

[17] Ioan Doré Landau, Abraham Castellanos-Silva, Tudor-Bogdan Airimiţoaie, Gabriel Buche, and Mathieu Noe. Benchmark on adaptive regulation - rejection of unknown/time-varying multiple narrow band disturbances. European Journal of Control, 2013.

[18] Gang Li. A stable and efficient adaptive notch filter for direct frequency estimation. Signal Processing, IEEE Transactions on, 45(8):2001 -2009, aug 1997.

[19] R. Marino, G.L. Santosuosso, and P. Tomei. Robust adaptive com- 


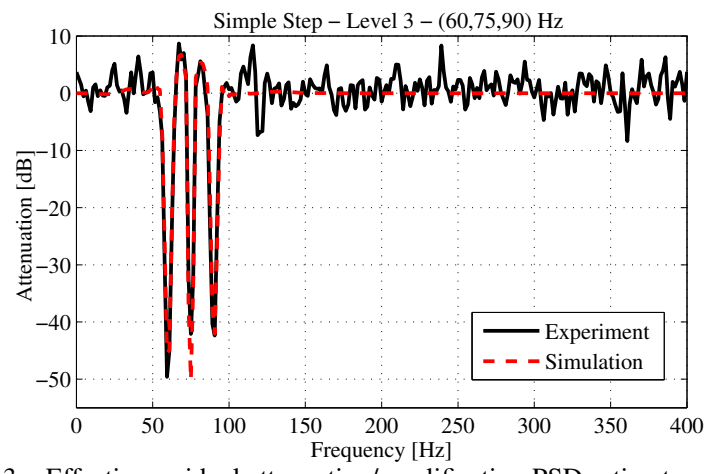

Fig. 13. Effective residual attenuation/amplification PSD estimates computed as the difference between the open loop PSD and the closed loop PSD Comparison between the simulation and the real-time result for the Simple Step Test with 3 narrow-band disturbances at 60,75 , and $90 \mathrm{~Hz}$.
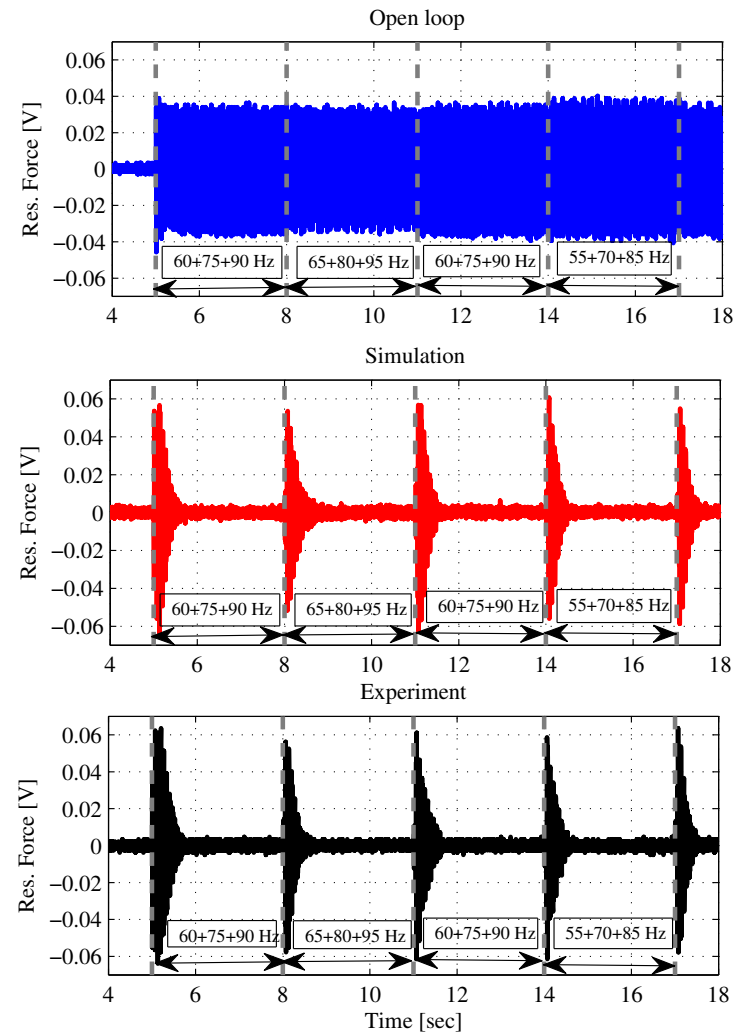

Fig. 14. Time response comparison between the open loop (top), simulation (middle), and the real-time results (bottom) for the Step Frequency Changes Test - Sequence 2.

pensation of biased sinusoidal disturbances with unknown frequency. Automatica, 39:1755-1761, 2003.

[20] Riccardo Marino, Giovanni L. Santosuosso, and Patrizio Tomei. Output feedback stabilization of linear systems with unknown additive output sinusoidal disturbances. Eur. J. Control, 14(2):131-148, 2008.

[21] N.K. M'Sirdi, H.R. Tjokronegoro, and I.D. Landau. An rml algorithm for retrieval of sinusoids with cascaded notch filters. In Acoustics, Speech, and Signal Processing, 1988. ICASSP-88., 1988 International Conference on, pages $2484-2487$ vol.4, apr 1988.

[22] A. Nehorai. A minimal parameter adaptive notch filter with constrained poles and zeros. IEEE Trans. Acoust., Speech, Signal Processing, ASSP33:983-996, 1985.

[23] G. Obregon-Pulido, B. Castillo-Toledo, and A. Loukianov. A globally convergent estimator for $\mathrm{n}$-frequencies. IEEE Trans. Autom. Control, 47(5):857-863, 2002.

[24] H. Prochzka and I.D. Landau. Pole placement with sensitivity function shaping using 2nd order digital notch filters. Automatica, 39(6):1103 1107,2003

[25] Anton A. Pyrkin. Adaptive algorithm to compensate parametrically

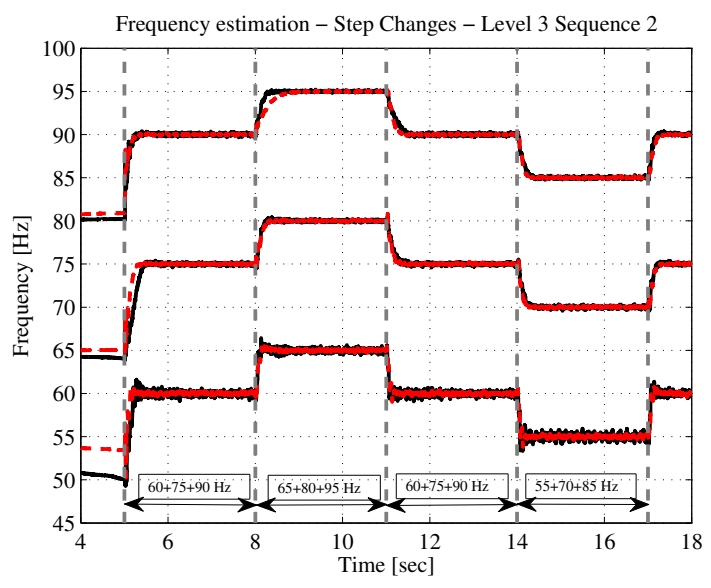

Fig. 15. Frequency estimation for the sequence 2 in real time (solid line) and in simulation (dashed line).
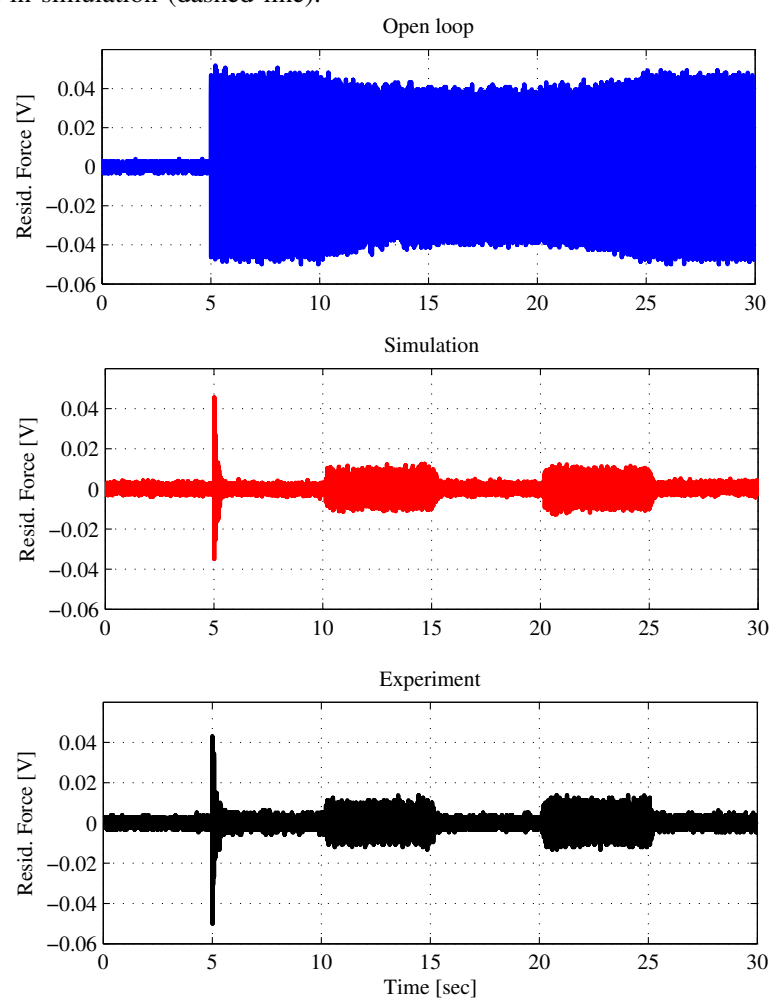

Fig. 16. Time response comparison between the open loop (top), simulation (middle), and real-time (bottom) results for the Chirp Test.

uncertain biased disturbance of a linear plant with delay in the control channel. Automation and Remote Control, 71(8):1562-1577, 2010.

[26] D.B. Rao and Sun-Yuan Kung. Adaptive notch filtering for the retrieval of sinusoids in noise. Acoustics, Speech and Signal Processing, IEEE Transactions on, 32(4):791 - 802, aug 1984.

[27] Phillip A. Regalia. An improved lattice-based adaptive iir notch filter Signal Processing, IEEE Transactions on, 9(9):2124 - 2128, september 1991.

[28] Petre Stoica and Arye Nehorai. Performance analysis of an adaptive notch filter with constrained poles and zeros. IEEE Trans. Acoust. Speech, Signal Processing, 36(6):911 - 919, 1988.

[29] T.T. Tay, I.M.Y. Mareels, and J.B. Moore. High Performance Control. Birkh auser Boston, 1997.

[30] Petr Tichavský and Arye Nehorai. Comparative study of four adaptive frequency trackers. Automatic Control, IEEE Transactions on, 45(6): 1473 - 1484, 1997.

[31] Y.Z. Tsypkin. Stochastic discrete systems with internal models. Journal of Automation and Information Sciences, 29(4\&5):156-161, 1997. 